УДК 538.915

\title{
КРЕМНИЙ-НИОБИЕВЫЕ БЛОКИ ДЛЯ КОНСТРУИРОВАНИЯ НАНОСТРУКТУРИРОВАННЫХ МАТЕРИАЛОВ
}

\author{
(C2018 Н. А. Борщ ${ }^{1}$, Н. С. Переславцева ${ }^{1}$, С. И. Курганский ${ }^{2}$ \\ ${ }^{1}$ Воронежский государственный технический университет, Московский пр., 14, 394026 Воронеж, Россия \\ ${ }^{2}$ Воронежский государственный университет, Университетская пл., 1, 394018 Воронеж, Россия \\ e-mail:n.a.borshch@ya.ru
}

Поступила в редакцию 07.02.2018

\begin{abstract}
Аннотация. Представлены результаты компьютерного моделирования пространственной структуры анионных кремний-ниобиевых кластеров из 10, 12, 14 и 16 атомов кремния. Для каждого кластера представлен ряд стабильных изомеров с качественно различной структурой, которые могут служить элементарными блоками для построения более крупных наноструктурированных объектов - нанопроволок, нанопроводов, нанокристаллов.
\end{abstract}

Ключевые слова: теория функционала плотности, атомные кластеры, электронная структура, наноматериалы.

DOI: https://doi.org/10.17308/kcmf.2018.20/511

\section{ВВЕДЕНИЕ}

Одной из самых актуальных задач современной науки является поиск качественно новых материалов, которые помогли бы решить задачу миниатюризации электронных устройств. Несомненно, самыми перспективными объектами с этой точки зрения являются структуры пониженной размерности - нольмерные (кластеры) и одномерные (нанотрубки, нанопровода). В 1985 году, когда был получен первый углеродный фуллерен [1], появились предположения, что и кремний может образовывать похожие структуры. Первые кремниевые кластеры с замкнутой структурой были синтезированы в 2001 году [2]. Стабильность кремниевой «клетки» обеспечивает инкапсулированный атом металла. Оказалось, что сорт атома металла влияет и на свойства кластера, определяя особенности атомной структуры и электронно-энергетического спектра [3-6]. Благодаря своей структуре, некоторые кремниевые кластеры могут служить строительными единицами для построения более крупных наноструктур (например, нанотрубок и нанокристаллов) с заранее заданными свойствами [7-9].

Кластеры $\mathrm{MeSi}_{10}^{-}$и $\mathrm{MeSi}_{12}^{-}$(Me - атом металла) могут иметь структуру призмы или антипризмы, центрированной атомом металла, благодаря чему они рассматриваются как возможные элементарные блоки для построения более крупных нанокластеров и кремний-металлических нанопро- волок [7-9]. Кластеры $\mathrm{MeSi}_{16}^{-}$могут иметь фуллереноподобную структуру, что позволит использовать их для формирования нанокристаллов по типу кремниевых [10-12] и германиевых [13] клатратных кристаллов.

В данной работе представлены результаты компьютерного моделирования пространственной структуры анионных кремний-ниобиевых кластеров из $10,12,14$ и 16 атомов кремния. Для каждого кластера представлен ряд стабильных изомеров с качественно различной структурой, которые могут служить элементарными блоками для построения более крупных наноструктурированных объектов нанопроволок, нанопроводов, нанокристаллов.

\section{ДЕТАЛИ РАСЧЕТА}

Расчеты проводились методом функционала плотности (B3LYP [14]) в комбинированном базиce 6-311+G(d) [15] для атомов кремния и DGDZVP [16] для атома ниобия с использованием пакета программ Gaussian 09 [17]. В результате расчета получались собственные значения энергии каждой молекулярной орбитали, т.е. энергетический спектр, в котором каждую молекулярную орбиталь можно представить в виде уровня. Теоретические спектры получались после того, как каждый энергетический уровень заменялся гауссовым распределением с полушириной 0.15 эВ и интенсивности всех распределений при каждом значении энер- 
гии складывались. Совмещение рассчитанных и экспериментальных спектров по энергетической шкале проводилось по положению верхней заполненной орбитали.

Средняя энергия связи $E_{b}$ вычислялась по формуле:

$$
E_{b}=\frac{n E(\mathrm{Si})+E^{-}(\mathrm{Nb})-E\left(\mathrm{NbSi}_{n}^{-}\right)}{n+1},
$$

где $E(\mathrm{Si})$ и $E^{-}(\mathrm{Nb})$ - полные энергии свободного атома кремния и аниона ниобия, соответственно, $E\left(\mathrm{NbSi}_{n}^{-}\right)$- полная энергия кластера, $n-$ число атомов кремния в кластере.

\section{РЕЗУЛЬТАТЫ И ИХ ОБСУЖДЕНИЕ}

На рис. 1 показаны структуры изомеров кластеров.

Основной изомер $\mathrm{NbSi}_{10}^{-}(\mathrm{A})$ имеет структуру пятиугольной бипирамиды с атомом ниобия в одной из вершин и с четырьмя дополнительными атомами кремния. Атом ниобия образует связи $\mathrm{Nb}-\mathrm{Si}$ длинами от 2.68 до $2.90 \AA$ со всеми атомами кремния. Наименьшая длина связи Si-Si в этой структуре - между дополнительными атомами кремния и составляет $2.33 \AA$, наибольшая длина связи между атомами кремния - в основании бипирамиды и составляет $2.83 \AA$. Основное состояние изомера синглетное.

Изомер $\mathrm{NbSi}_{10}^{-}(\mathrm{B})$ получился в результате оптимизации кластера с исходной структурой правильной пятиугольной призмы, центрированной атомом ниобия. В результате оптимизации по одной связи между соседними атомами кремния в каждом основании призмы оказались значительно ослаблены - межатомные расстояния $\mathrm{Si}-\mathrm{Si}$ для них составляют $4.00 \AA$. Длины связей $\mathrm{Nb}-\mathrm{Si}$ составляют $2.61,2.74$ или $2.77 \AA$. Атом ниобия формирует связи со всеми атомами кремния. Длины связей $\mathrm{Si}-\mathrm{Si}$ для атомов из одного основания зависят от их близости к атому ниобия - связи между ближними к нему атомами составляют $2.35 \AA$, а между более удаленными - $2.39 \AA$. Связи между атомами кремния из разных оснований имеют длины $2.38,2.56$ или $2.63 \AA$. Основное состояние кластера - синглетное. В триплетном и квинтетном состояниях в результате оптимизации были получены менее стабильные и значительно более искаженные призмоподобные структуры.

Изомер $\mathrm{NbSi}_{10}^{-}(\mathrm{C})$ имеет продольную структуpy, состоящую из двух искаженных октаэдров с общей вершиной, в которой расположен атомом ниобия. Атом ниобия образует связи с восемью ато- мами кремния - четыре длинами по $2.68 \AA$ и четыре по $2.76 \AA$ А. Связи Si-Si в этой структуре имеют длины $2.35,2.41$ и $2.69 \AA$. Основное состояние изомера - триплетное. Средняя энергия связи в синглетном состоянии лишь на 0.0006 эВ/атом меньше, чем в триплетном, поэтому можно предположить возможность существования кластера и в этом спиновом состоянии. Структура кластера в синглетном состоянии идентична структуре основного состояния, отличия в межатомных расстояниях составляют $\sim 0.01 \AA$.

Изомер $\mathrm{NbSi}_{10}^{-}$(D) имеет структуру пятиугольной антипризмы, центрированной атомом ниобия. Атом ниобия формирует связи длинами по $2.57 \AA$ со всеми атомами кремния. Расстояния между ближайшими атомами кремния из разных оснований составляют $2.87 \AA$, а в одном основании $-2.64 \AA$. Изомер со структурой антипризмы стабилен только в синглетном состоянии, его оптимизация в триплетном и квинтетном состояниях привела к низкосимметричным структурам.

Исходной конфигурацией для оптимизации основного изомера $\mathrm{NbSi}_{12}^{-}(\mathrm{A})$ служила центрированная атомом ниобия правильная шестиугольная призма. В результате оптимизации форма кластера сохранилась. Расстояния Si-Si в основаниях призмы одинаковы и составляют $2.42 \AA$, расстояние между основаниями $2.45 \AA$. Межатомные расстояния $\mathrm{Nb}-\mathrm{Si}$ одинаковы для всех атомов кремния и составляют $2.71 \AA$ А. Изомер со структурой призмы наиболее стабилен в синглетном состоянии (средняя энергия связи составляет 3.62 эВ/атом), оптимизированные в триплетном и квинтетном состоянии структуры значительно менее стабильны. В триплетном и квинтетном состояниях структура изомера также призма, но ее геометрия значительно изменяется, призма искажается, средние энергии связи существенно ниже -3.50 в триплетном состоянии и 3.41 эВ/атом - в квинтетном.

В результате оптимизации кластера со структурой антипризмы был получен изомер $\mathrm{NbSi}_{12}^{-}(\mathrm{B})$. Межатомные расстояния $\mathrm{Si}-\mathrm{Si}$ для атомов из одного основания одинаковы и составляют $2.48 \AA$, pacстояние между ближайшими атомами кремния из разных оснований $-2.61 \AA$. Атом ниобия образует связи одинаковой длины $2.73 \AA ̊$ со всеми атомами кремния. Синглетное состояние кластера $\mathrm{NbSi}_{12}^{-}$(В) является основным. В триплетном и квинтетном состоянии структура качественно не меняется, но средние энергии связи этих структур существенно ниже -3.47 и 3.41 эВ/атом против 3.54 эВ/атом в основном изомере. 

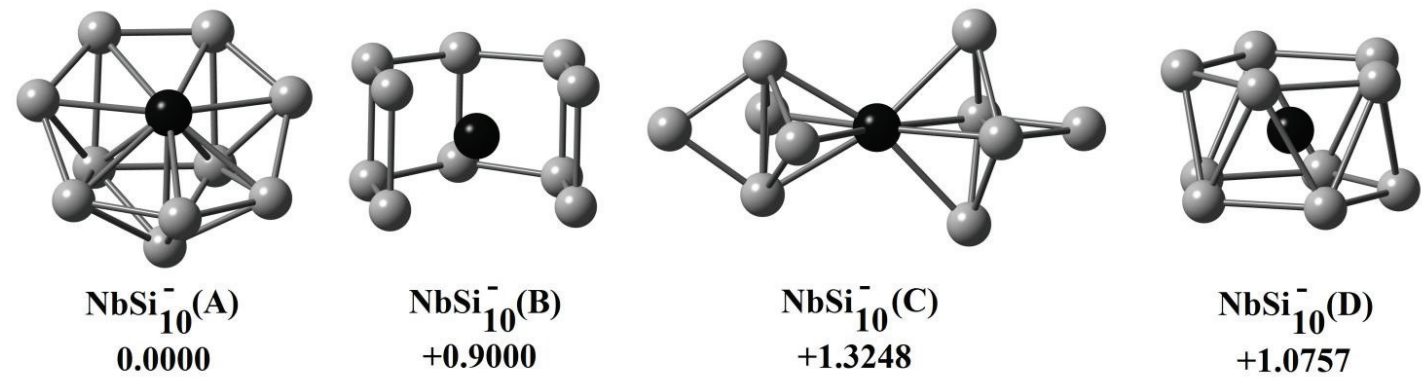

$$
\begin{gathered}
\mathrm{NbSi}_{10}^{-}(\mathrm{C}) \\
+\mathbf{1 . 3 2 4 8}
\end{gathered}
$$

$$
\begin{gathered}
\mathrm{NbSi}_{10}^{-}(\mathrm{D}) \\
+\mathbf{1 . 0 7 5 7}
\end{gathered}
$$
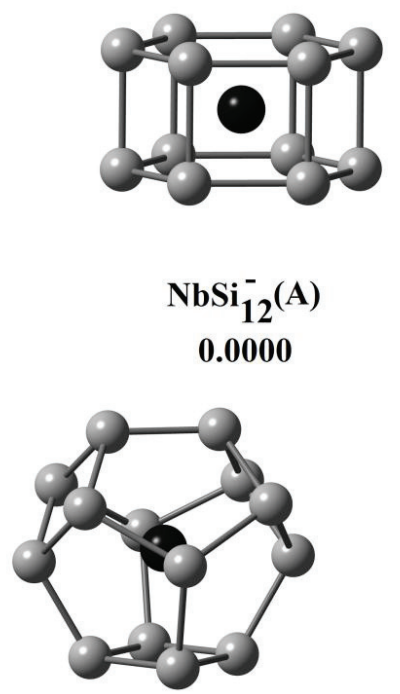

$$
\begin{gathered}
\mathrm{NbSi}_{14}^{-}(\mathrm{A}) \\
\mathbf{0 . 0 0 0 0}
\end{gathered}
$$
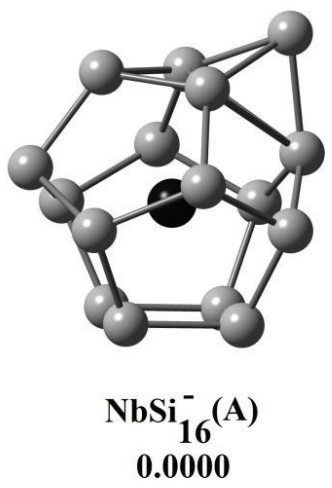

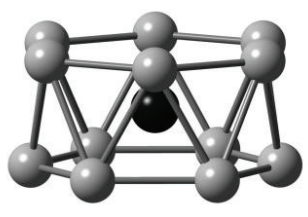

$$
\mathrm{NbSi}_{12}^{-}(\mathrm{B})
$$$$
+0.9863
$$

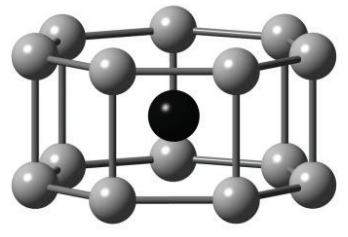

$$
\begin{gathered}
\mathrm{NbSi}_{14}^{-}(\mathrm{B}) \\
+\mathbf{1 . 9 9 0 0}
\end{gathered}
$$

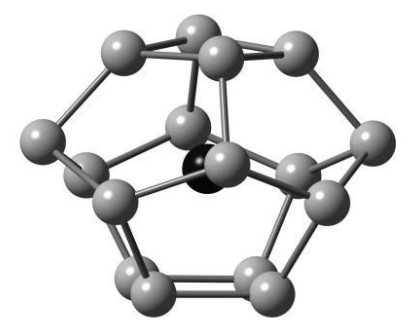

$$
\begin{gathered}
\mathrm{NbSi}_{16}^{-}(\mathrm{B}) \\
+0.6421
\end{gathered}
$$

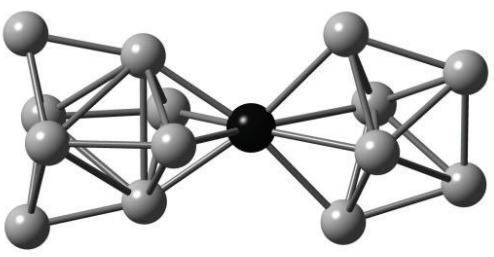

$$
\begin{gathered}
\mathrm{NbSi}_{\mathbf{1 4}}^{-}(\mathrm{C}) \\
+\mathbf{3 . 1 3 8 7}
\end{gathered}
$$

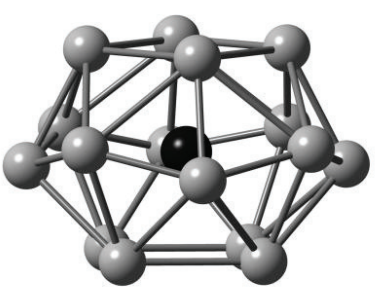

$$
\begin{gathered}
\mathrm{NbSi}_{16}^{-}(\mathrm{C}) \\
+3.1562
\end{gathered}
$$

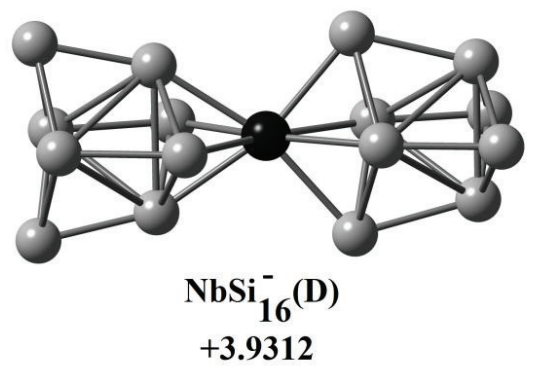

Рис. 1. Пространственные структуры кластеров $(n=10,12,14,16)$. Черным цветом показан атом ниобия. Числа указывают разницу между полной энергией в основном изомере и побочных (в эВ)

[Fig. 1. Geometric structures of $\mathrm{NbSi}_{\mathrm{n}}^{-}(n=10,12,14,16)$ clusters. Black ball - niobium atom, grey balls - silicon atoms Numbers indicate the difference between the total energies of an isomers B, C and D from the isomer A (in eV)] 


\section{Н. А. БОРЩ, Н. С. ПЕРЕСЛАВЦЕВА, С. И. КУРГАНСКИЙ}

Еще один изомер кластера $\mathrm{NbSi}_{12}^{-}$, структура которого может оказаться перспективной для построения более крупных нанообъектов, - изомер $\mathrm{NbSi}_{12}^{-}(\mathrm{C})$ с продольной структурой из двух звеньев, соединенных атомом ниобия. Структура этого изомера построена из двух пятиугольных бипирамид с атомом ниобия в основании. Атом ниобия образует восемь связей $\mathrm{Nb}-\mathrm{Si}$ : четыре связи длиной $2.79 \AA$ с атомами кремния из вершин бипирамид и четыре связи длиной $2.67 \AA-$ с атомами кремния из их оснований. Длины связей $\mathrm{Si}-\mathrm{Si}$ в этом кластере составляют от 2.45 до $2.59 \AA$. Основное состояние изомера $\mathrm{NbSi}_{12}^{-}(\mathrm{C})$ - триплетное $\left(E_{\mathrm{b}}=3.42\right.$ эВ/атом). В синглетном состоянии средняя энергия связи лишь на 0.001 эВ/атом меньше, чем в триплетном, а пространственная структура мало отличается от структуры в основном состоянии. Квинтетное состояние существенно менее стабильно - ему соответствует средняя энергия связи 3.34 эВ/атом.

Основной изомер $\mathrm{NbSi}_{14}^{-}(\mathrm{A})$ - многогранник с шестью пятиугольными и тремя четырехугольными гранями, который можно назвать фуллереноподобным. Атом ниобия формирует связи длинами $2.68,2.73$ или $2.87 \AA$ со всеми атомами кремния. Межатомные расстояния $\mathrm{Si}-\mathrm{Si}$ в этом изомере имеют значения 2.35 и $2.43 \AA$. Основное состояние этого кластера - синглетное. Изомер $\mathrm{NbSi}_{14}^{-}(\mathrm{B})$ представляет собой правильную семиугольную призму, центрированную атомом ниобия. Расстояния между ближайшими атомами кремния в одном основании составляют $2.35 \AA$, а между ближайшими атомами кремния из разных оснований $-2.46 \AA$. Атом ниобия формирует связи длинами $2.97 \AA$ со всеми атомами кремния. Изомер $\mathrm{NbSi}_{14}^{-}(\mathrm{C})$ имеет продольную структуру из двух звеньев с общим атомом ниобия. Одно звено представляет собой пятиугольную бипирамиду с атомом ниобия в основании, второе - аналогичную бипирамиду с двумя дополнительными атомами кремния. Атом ниобия образует восемь связей $\mathrm{Nb}-\mathrm{Si}$ длинами 2.56-2.78 А. Длины связей между атомами кремния в этом кластере составляют $2.41-2.94 \AA$. Основное состояние этого кластера - синглетное.

При оптимизации кластера со стартовой структурой многогранника Франка-Каспера, центрированного атомом ниобия, в синглетном состоянии был получен основной изомер кластера $\mathrm{NbSi}_{16}^{-}$ . Многогранник Франка-Каспера трансформировался в фуллереноподобную искаженную структуpy, и основной изомер $\mathrm{NbSi}_{16}^{-}(\mathrm{A})$ представляет со- бой многогранник с двумя четырехугольными основаниями и пятиугольными боковыми гранями. Один из атомов кремния в основании многогранника находится на существенно большем расстоянии от атома ниобия, чем остальные - $4.11 \AA \AA$ против 2.32-2.67 А. Оптимизация кластера со структурой многогранника Франка-Каспера в триплетном и квинтетном состояниях также привела к его трансформации в фуллереноподобные многогранники, но средняя энергия связи в них существенно ниже, чем в синглетном состоянии.

В результате оптимизации стартовой структуры фуллереноподобного многогранника, центрированного атомом ниобия, был получен фуллереноподобный изомер $\mathrm{NbSi}_{16}^{-}(\mathrm{B})$. Для двух атомов кремния на боковой поверхности многогранника расстояния $\mathrm{Nb}-\mathrm{Si}$ составляют $3.29 \AA$, для остальных они составляют от 2.83 до $2.95 \AA$ А. Длины связей между атомами кремния в этой структуре составляют 2.28-2.52 А̊. При оптимизации фуллереноподобного многогранника в триплетном состоянии получается менее деформированный многогранник - для двух наиболее удаленных от атома ниобия атома кремния расстояния $\mathrm{Nb}-\mathrm{Si}$ составляют только $3.05 \AA$ А. Средняя энергия связи этого изомера незначительно меньше, чем изомера в синглетном сотстоянии, поэтому нельзя исключать возможность его существования.

Структура изомера $\mathrm{NbSi}_{16}^{-}(\mathrm{C})$ - многогранник с двумя квадратными основаниями и треугольными боковыми гранями. Длины связей Si-Si в основаниях равны $2.70 \AA$ А. Боковые грани представляют собой треугольники двух типов - равнобедренные с двумя сторонами длинами по $2.76 \AA$ и одной длиной $2.70 \AA$ и неправильные со сторонами длинами $2.39,2.49$ и $2.76 \AA$. Атом ниобия формирует восемь связей длинами по $2.74 \AA$ с атомами кремния в основаниях многогранника. Межатомные расстояния $\mathrm{Nb}-\mathrm{Si}$ с атомами боковой поверхности существенно больше $-3.06 \AA$.

Изомер $\mathrm{NbSi}_{16}^{-}$(D) имеет продольную структуру, построенную из двух звеньев, аналогичным большим звеньям в кластерах $\mathrm{NbSi}_{14}^{-}(\mathrm{C})$. Атом ниобия образует восемь связей $\mathrm{Nb}-\mathrm{Si}$ - четыре длинами по 2.62 А и четыре длинами по $2.63 \AA$. Длины связей между атомами кремния составляют 2.42$2.92 \AA$. Основное состояние изомера - триплетное. Структуры кластера в синглетном и квинтетном состоянии идентичны и лишь незначительно отличаются количественно.

Сравнение рассчитанных данных о структуре кластеров с экспериментальными не представля- 
ется возможным, поскольку современное состояние экспериментальных техник изучения геометрических структур нанообъектов не позволяет исследовать кластеры таких размеров. Один из самых надежных способов подтвердить адекватность оптимизированных структур является сопоставление их рассчитанных электронных спектров с экспериментальными фотоэлектронными [18]. На рис. 2 показано сопоставление рассчитанных электронных спектров с экспериментальными фотоэлектронными спектрами [18]. Рассчитанные электронный спектр основных изомеров кластеров $\mathrm{NbSi}_{n}^{-}$хорошо согласуются с экспериментальными [18], что позволяет полагать, что экспериментально были детектированы именно эти изомеры. Имеющиеся небольшие различия в теоретических и экспериментальных спектрах вполне объяснимы присутствием в экспериментальном ансамбле нанокластеров небольшой доли неосновных изомеров.

\section{ЗАКЛЮЧЕНИЕ}

Для анионных кремний-ниобиевых кластеров возможно формирование двух типов структур, которые могут служить элементарными блоками для конструирования более крупных наноформ - эндоэдральные и продольные структуры. Эндоэдральные структуры более стабильны и сравнение их рассчитанных электронных спектров с экспериментальными фотоэлектронными свидетельствует о том, что именно они были детектированы экспериментально.

Для представленных расчетов были использованы вычислительные ресурсы Суперкомпьютерного иентра Воронежского государственного университета.
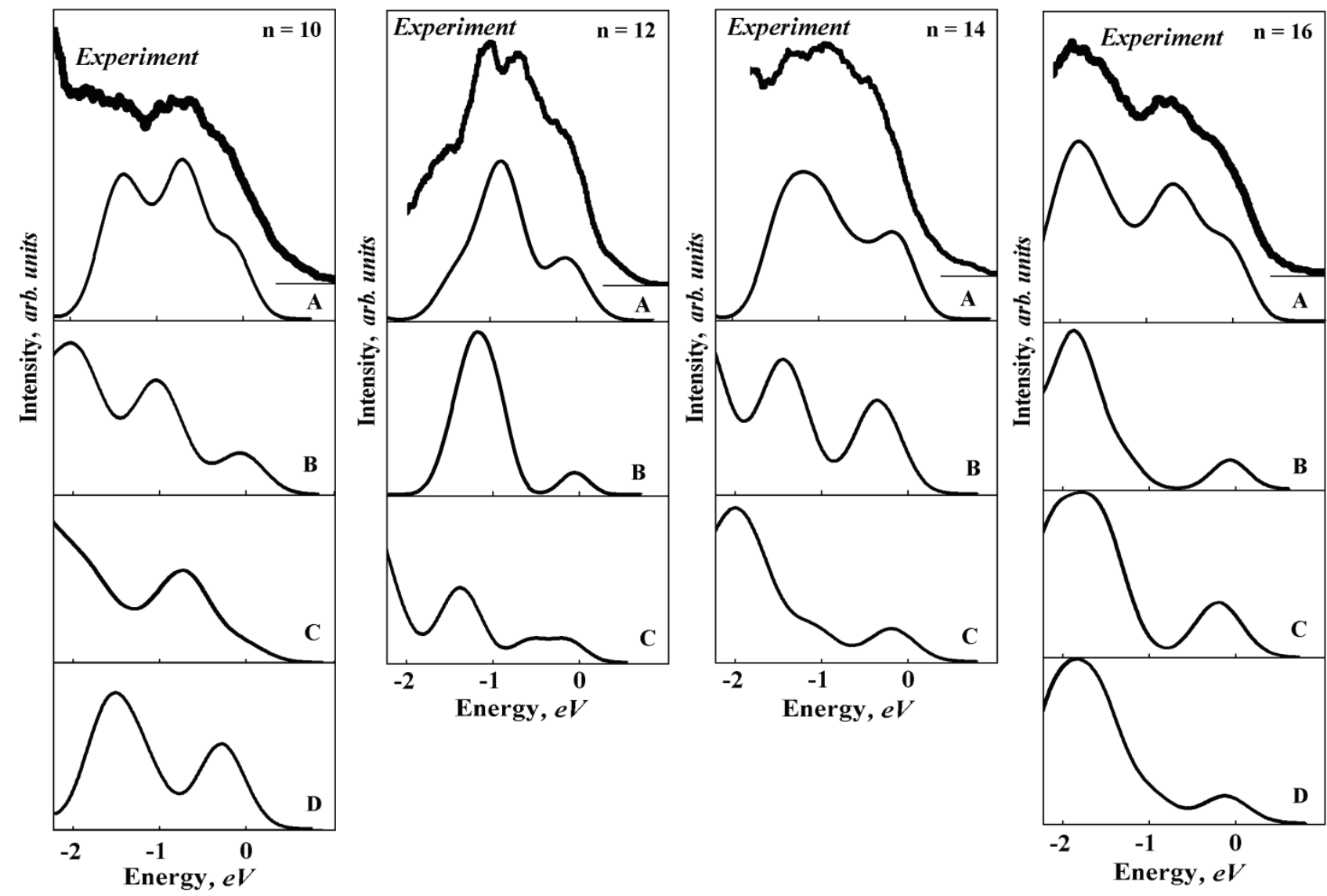

Рис. 2. Сопоставление рассчитанных плотностей электронных состояний кластеров $(n=10,12,14,16)$ с экспериментальными фотоэлектронными спектрами [18]. За начало отсчета по энергетической шкале принято положение верхней заполненной орбитали. Жирными линиями показаны экспериментальные спектры

[Fig. 2. Comparison of the calculated electron spectra of $\mathrm{NbSi}_{n}^{-}(n=10,12,14,16)$ with experimental photoelectron spectra [18]. The location of the higher occupied molecular orbital is taken as the origin of counting] 


\section{СПИСОК ЛИТЕРАТУРЫ}

1. Kroto H. W., Heath J. R., O’Brien S. C., Curl R. F. Smalley R. E. // Nature, 1985, vol. 318, pp. 162-163. DOI: $10.1038 / 318162 \mathrm{a} 0$

2. Hiura H., Miyazaki T., Kanayama T. // Phys. Rev. Lett., 2001, vol. 86, pp. 1733-1736. DOI: 10.1103/PhysRevLett.86.1733

3. Reveles J. U., Khanna S. N. // Phys. Rev. B, 2006, vol. 74(3), pp. 035435-035440. DOI: 10.1103/PhysRevB.74.035435

4. Guo L., Zhao G., Gu Y., Liu X., Zeng Z. // Phys. Rev. $B$, 2008, vol. 77, pp. 195417-195424. DOI: 10.1103/PhysRevB.77.195417

5. Borshch N., Kurganskii S. // Journal of Applied Physics, 2014, vol. 116, № 12, pp. 124302-1-124302-8. DOI: $10.1063 / 1.4896528$

6. Борщ Н. А., Переславцева Н. С., Курганский С. И. // $\Phi T T, 2014$, т. 56, № 11 , с. 2259-2264. DOI: $10.1134 /$ S1063783414110055

7. Palagin D., Reuter K. // ACS Nano, 2013, vol. 7 (2), pp. 1763-1768. DOI: $10.1021 / \mathrm{nn} 3058888$

8. Lu A. // Journal of Modern Physics, 2013, vol. 4, pp. 501-504. DOI: 10.4236/jmp.2013.44071

9. Mpourmpakis G., Froudakis G. E., Andriotis A. N., Menon M. // Phys. Rev. B, 2003, vol. 68, pp. 125407-1125407-5. DOI: 10.1103/PhysRevB.68.125407
10. Kasper J. S., Hagenmuller P., Pouchard M., Cros C. // Science, 1965, vol. 150, pp. 1713-1716. DOI: 10.1126/science.150.3704.1713

11. Курганский С. И., Борщ Н. А., Переславцева Н. С. // ФТП, 2005, т. 39, № 10, с. 1218-1223. DOI: $10.1134 / 1.2085266$

12. Борщ Н. А., Переславцева Н. С., Курганский С. И. // ФТП, 2011, т. 45, № 6, с. 729-739. DOI: $10.1134 / \mathrm{S} 1063782611060066$

13. Борщ Н. А., Переславцева Н. С., Курганский С. И. // ФТП, 2009, т. 43, № 5, с. 590-594. DOI: $10.1134 / \mathrm{S} 106378260$

14. Lee C., Yang W., Parr R. G. // Phys. Rev. B, 1988, vol. 37, pp. 785-789. DOI: 10.1103/PhysRevB.37.785

15. Wachters A. J. H. // J. Chem. Phys., 1970, vol. 52, pp. 1033-1036. DOI: 10.1063/1.1673095

16. Godfrey S. M., Hinchliffe A. // J. Mol. Struct. Theochem, 2006, vol. 761, pp. 1-5. DOI: 10.1016/j.theochem.2005.11.029

17. Gaussian 09, Revision B09; Gaussian, Inc: Pittsburg, PA, 2009.

18. Koyasu K., Atobe J., Furuse S., Nakajima A. // J. Chem. Phys., 2008, vol. 129, pp. 214301-214307. DOI: $10.1039 / \mathrm{c} 2 \mathrm{cp} 23247 \mathrm{~b}$

\title{
SILICON-NIOBIUM BLOCKS FOR THE DESIGNING OF NEW NANOSTRUCTURES
}

\author{
C)2018 N. A. Borshch ${ }^{1}$, N. S. Pereslavtseva ${ }^{1}$, S. I. Kurganskii ${ }^{2}$ \\ ${ }^{1}$ Voronezh State Technical University, 14 Moskovskii av., 394026 Voronezh, Russia \\ ${ }^{2}$ Voronezh State University, 1 Universitetskaya pl., 394018 Voronezh Russia \\ e-mail:n.a.borshch@ya.ru
}

Received 03.10.2017

\begin{abstract}
This paper presents the calculation results of the spatial structure and electron energy spectrum of the anionic silicon-niobium clusters $\mathrm{NbSi}_{n}^{-}(n=10,12,14,16)$. A comparison of the calculated total density of electronic states with experimental photoelectron spectra allowed us to determine the structure of the clusters. The calculations were carried out within the density functional theory (DFT) framework where the Becke's three-parameter and Lee-Yang-Parr's gradientcorrected correlation hybrid functional (B3LYP) and 6-311+G(d) basis sets were used for the silicon atoms and DGDZVP basis sets were used for the niobium atom for a structural optimization as implemented in GAUSSIAN 09 (G09).

The major isomer of the $\mathrm{NbSi}_{10}^{-}$cluster is a pentagonal bipyramid with a niobium atom in one of the vertices and with four additional silicon atoms. The major isomer of the $\mathrm{NbSi}_{12}^{-}$cluster is a distorted hexagonal prism with an incapsulated niobium atom. The hexagonal anti-prism isomer and the prolate isomer are also stable. The segment of the prolate isomer are pentagonal bipyramids, with a shared niobium atom at the basis. The major isomer of the $0 \mathrm{NbSi}_{14}^{-}$cluster is a fullerene-like polyhedron with an encapsulated niobium atom. The prolate isomer of this cluster is also stable, but has a lower average binding energy than prismatic isomers. The major isomer of the $\mathrm{NbSi}_{16}^{-}$cluster has a fullerene-like structure in the form of a polyhedron with eight pentagonal side edges and rhombic bases. The prolate isomer of this cluster is also stable, but has a lower average binding energy than prismatic isomers.
\end{abstract}


Thus, two types of structures possible for anionic silicon-niobium clusters are endohedral and prolate structures. Endohedral structures are more stable and their calculated electron spectra are more consistent with experimental photoelectron spectra.

Keywords: DFT, atomic clusters, electronic structure, nanostructures.

DOI: https://doi.org/10.17308/kcmf.2018.20/511

\section{ACKNOWLEDGEMENTS}

The presented calculations were made with the help of the computing resources of the Supercomputer Centre of Voronezh State University.

\section{REFERENCES}

1. Kroto H. W., Heath J. R., O’Brien S. C., Curl R. F. Smalley R. E. Nature, 1985, vol. 318, pp. 162-163. DOI: $10.1038 / 318162 \mathrm{a} 0$

2. Hiura H., Miyazaki T., Kanayama T. Phys. Rev. Lett., 2001, vol. 86, pp. 1733-1736. DOI: 10.1103/PhysRevLett.86.1733

3. Reveles J. U., Khanna S. N. Phys. Rev. B, 2006, vol. 74(3), pp. 035435-035440. DOI: 10.1103/PhysRevB.74.035435

4. Guo L., Zhao G., Gu Y., Liu X., Zeng Z. Phys. Rev. B, 2008, vol. 77, pp. 195417-195424. DOI: 10.1103/PhysRevB.77.195417

5. Borshch N., Kurganskii S. Journal of Applied Physics, 2014, vol. 116, no. 12, pp. 124302-1-124302-8. DOI: $10.1063 / 1.4896528$

6. Borshch N. A., Pereslavtseva N. S., Kurganskii S. I. Physics of the Solid State, 2014, vol. 56, no. 11, pp. 23362342. DOI: $10.1134 / \mathrm{S} 1063783414110055$

7. Palagin D., Reuter K. ACS Nano, 2013, vol. 7 (2), pp. 1763-1768. DOI: $10.1021 / \mathrm{nn} 3058888$

8. Lu A. Journal of Modern Physics, 2013, vol. 4, pp. 501-504. DOI: 10.4236/jmp.2013.44071
9. Mpourmpakis G., Froudakis G. E., Andriotis A. N., Menon M. Phys. Rev. B, 2003, vol. 68, pp. 125407-1125407-5. DOI: 10.1103/PhysRevB.68.125407

10. Kasper J. S., Hagenmuller P., Pouchard M., Cros C. Science, 1965, vol. 150, pp. 1713-1716. DOI: 10.1126/science. 150.3704 .1713

11. Kurganskii S. I., Borshch N. A., Pereslavtseva N. S. Semiconductors, 2005, vol. 39, no. 10, pp. 1176-1181. DOI: $10.1134 / 1.2085266$

12. Borshch N. A., Pereslavtseva N. S., Kurganskii S. I. Semiconductors, 2011, vol. 45, no. 6, pp. 713-723. DOI: 10.1134/S1063782611060066

13. Borshch N.A., Pereslavtseva N.S., Kurganskii S.I. Semiconductors, 2009, vol. 43, no. 5, pp. 563-567. DOI: $10.1134 /$ S106378260

14. Lee C., Yang W., Parr R. G. Phys. Rev. B, 1988, vol. 37, pp. 785-789. DOI: 10.1103/PhysRevB.37.785

15. Wachters A. J. H. J. Chem. Phys., 1970, vol. 52, pp. 1033-1036. DOI: 10.1063/1.1673095

16. Godfrey S. M., Hinchliffe A. J. Mol. Struct. Theochem, 2006, vol. 761, pp. 1-5. DOI: 10.1016/j.theochem.2005.11.029

17. Gaussian 09, Revision B09; Gaussian, Inc: Pittsburg, PA, 2009.

18. Koyasu K., Atobe J., Furuse S., Nakajima A. J. Chem. Phys., 2008, vol. 129, pp. 214301-214307. DOI: $10.1039 / \mathrm{c} 2 \mathrm{cp} 23247 \mathrm{~b}$
Бори Надежда Алексеевна - к. ф.-м. н., доцент, кафедра высшей математики и физико-математического моделирования, Воронежский государственный технический университет; тел.:+7(473) 2464222, e-mail: n.a.borshch@ya.ru

Переславиева Наталья Сергеевна - к. ф.-м. н., доцент, кафедра прикладной математики и механики, Воронежский государственный технический университет; тел.:+7(473) 2545475, e-mail: nsper@ya.ru

Курганский Сергей Иванович - д. ф.-м. н., професcop, кафедра физики твердого тела и наноструктур, Воронежский государственный университет; тел.:+7(473) 2208363, e-mail: kurganskii@ phys.vsu.ru
Nadezda A. Borshch - Cand. Sci. (Phys.-Math.), Higher Mathematics and Physical and Mathematical Modelling Department, Voronezh State Technical University; tel.: +7(473) 2208363, e-mail: n.a.borshch@ ya.ru

Natalia S. Pereslavtseva - Cand. Sci. (Phys.Math.), Applied Mathematics and Mechanics Department, Voronezh State Technical University; tel.: +7(473) 2545475, e-mail: nsper@ya.ru

Sergey I. Kurganskii - Dr. Sci. (Phys.-Math.), Professor, Solid State Physic and Nanostructures Department, Voronezh State University; tel.: +7(473) 2208363, e-mail: kurganskii@phys.vsu.ru 